\title{
Performance Comparison of MPLS and ATM Based Networks
}

\author{
Adnan Shakeel Ahmed, Md Omair Ahmad \\ \{adnanshakeel@protonmail.com,omair4ahmad@gmail.com\} \\ Department of CSE, Jamia Hamdard, New Delhi.
}

\begin{abstract}
This paper represents the prime factors of MPLS over ATM method and compares results of delay variations. In this paper we will see how the MPLS will provide better results for media traffic over the ATM method. In Computer Networks, MPLS contributes high scalability so we will briefly analyze the working methodology of MPLS system. MPLS helps in removing the dependence on Layer 2 technology. Among all the challenges faced in the Network industry traffic routing is one the most important, traffic routing and traffic algorithm are equally important and need to be efficient. We also observe that MPLS outperforms ATM in terms of delay and response time.
\end{abstract}

Keywords: MPLS, ATM, Networks, Layers, bandwidth, Traffic, Media, Routing, Algorithm, Computer, Internet, Service, Providers, protocol, switching

\section{Introduction}

Internet has seen an enormous growth since the World Wide Web (www) first surfaced back in 1991, from being used by a small percentage of people, it was earlier used by people in the research community and then later on by large public networks. Internet Service users have an increasing demand forcing the (ISP) to improve the services and the quality being provided. (1)The contemporary advances especially in the real-time Multimedia applications have increased our needs, for eg. faster routing, higher bandwidth etc. The algorithm that provides simple routing for IP gives its best performance only when it is suited to the data applications. Hence the reason why voice and video traffics are transferred over MPLS as compared to ATM. MPLS does have a number of issues that have to be fixed before it is generalized. However, MPLS offers several useful techniques that can improve network operations.

The MPLS is able to offer a simpler mechanism for traffic engineering and multi service functionality with the added benefits of greater scalability. MPLS can be defined as a segment of the evolving Internet to reduce the complexity by combining 
Layer -2 switching and Layer-3 routing into a fully integrated solution for fast packet forwarding. The MPLS offers a simpler technique for traffic engineering and functioning of multiple services and it adds up the benefits like having greater scalability. MPLS can be considered a part of the ever changing Internet that reduces the complexity by combining the Network Layer and the Data Layer routing which in turn provides a fast packet forwarding solution

\section{Multi-Protocol Label Switching (MPLS)}

Telecommunication technologies have been persistently changing and it seems to be improving faster and faster than ever-before. Transporting and delivering all types of communication in the same network and access circuit has been the long held goal in the telecommunications business.

MPLS changes the network mode from a routed network to a switched network. It would normally forward packets on a step by step basis but in such a scenario certain paths are established from source to destination. The paths which are predetermined are also known as Label Switching Paths (LSPs).

Label Switching Routers (LSRs) form a Label Switched Network and they come in different types:

- Ingress Router: This router is placed at the entrance of the LSP and it is through this router only that normal IP traffic can reach into the MPLS LSP. The incoming router receives the IP traffic and decides whether it has to go through an LSP to reach its desired destination, this incoming router encapsulates the IP traffic with a MPLS header and forwards it to the next point.

- Transit Router: It is can be any router in the middle of the LSP. Analyzing the incoming interface the router then decides where the packet is coming from and the MPLS header encapsulated and decides where to send the packet further.

- Pen-Ultimate Router: The penultimate route is the second last router in an LSP, the pen-ultimate router is the router before the last point in an LSP, since the last point in an LSP does not need to forward the packet to another transit router, it does not require the MPLS header. It is responsible for removing the MPLS header before passing it onto the last point in the LSP, having the MPLS header removed by the penultimate router before passing it on to the Egress router is optional.

- Egress Router: This router is placed at the exit point of the LSP, it receives the traffic from the pen-ultimate router, it looks up the IP and forwards it using the normal IP routing. 
MPLS can handle packets with specific characteristics, certain forwarding equivalence class (FEC) are assigned when a packet enters in a network for the first time One of the problems faced by conventional routing is the ability to remap routes, with the help of MPLS packets which carry real time traffic, such as voice or video. These can be remapped easily to low latency routes.

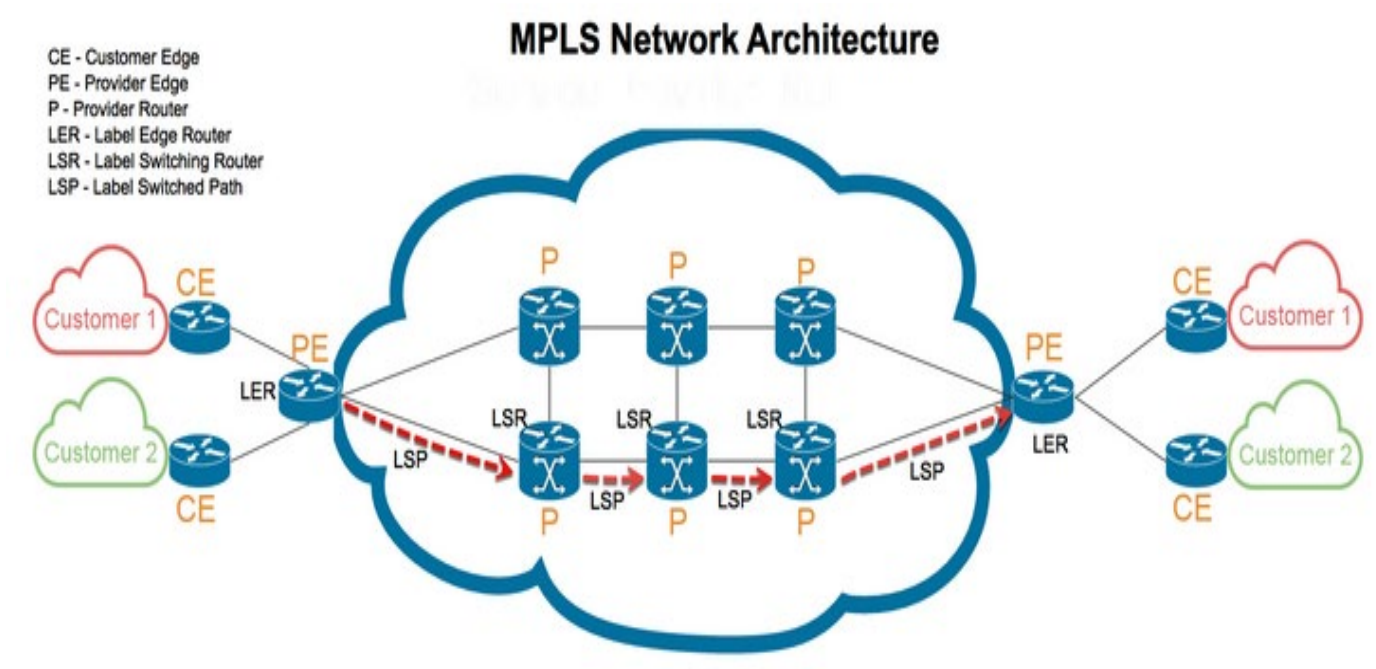

Fig. 1. MPLS Network Architecture

\section{ATM (Asynchronous Transfer Mode)}

Most technologies break data into small fragments that have poor performance because they lack the ability and mechanism to detect and transmit lost cells, when a piece of information is lost in a transit, the only way to recover it is through retransmitting the entire large packet again. ATM incorporates the technology to retransmit only the cells that were not transmitted properly. ATM routing depends on two connection types:

1. Virtual Channel Connection (VCC)

2. Virtual Path Connection (VPC). VPC is a collection of multiple VCCs.

ATM backbone involves legacy services and systems with corresponding ATM services and provides a base infrastructure for consolidation of networks.

The ATM implementation architecture that helps in accomplishing the integration of legacy system includes five-tier hierarchy. 
- An ATM backbone tier

- An ATM access tier

- A concentration tier

- An extended service tier

- A user tier

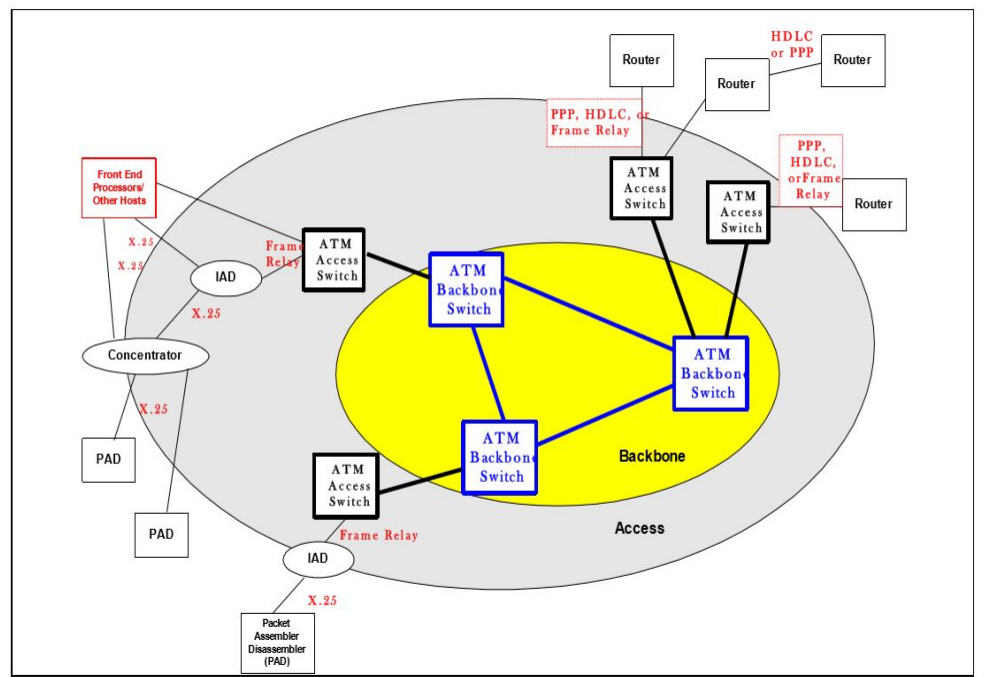

Fig. 2. ATM Backbone Architecture

\section{Why MPLS over ATM}

MPLS is a new technology which can be used over ATM by IP and can be setup in private networks as well as public networks which are maintained either by ISP or the Telecommunication Operators. It can be used for backbone networks which can be used for Internet Protocols as well as for other layer protocols. MPLS being an integrated model is an emerging IETF (Internet Engineering Task Force) standard architecture and is based on Tag switching of Cisco. (3)

The basic functionality of MPLS is to connect the power of Data Layer with the routing connectivity of Network Layer. Due to this the benefits of MPLS include reduced costs and reduced connectivity numbers as compared to ATM technology. A connection oriented mechanism is introduced in a connectionless IP network. Label Switched Path is established for each route or path through the network. 
ATM switching systems can have several structures with MPLS functionality which can exchange a label at packet forwarding engine.

MPLS was originally envisioned to overcome the four original problems:

1. Layer3 network layer routing scalability

2. Forwarding performances increases

3. Cell switched-based technologies integration

4. Increased routing services delivery flexibility

There are a few basic differences between MPLS and ATM, ATM works in a circuitswitched environment whereas, MPLS is made to work in modern packet switched networks which can be either Ethernet or even IP.

The difference becomes evident when the two types of network topologies are deployed. Point to Point connections are made in an ATM consisting of ATM adapters at the end of the physical circuit. On the other hand MPLS works similar to that of an Ethernet switch, it allows the network end points in any topology to be connected to the MPLS network and mesh with a particular customer's virtual mesh.

In order to recreate this design of meshing by the ATM, multiple ATM connections need to be installed at each organization's locations. The MPLS technology also enables the multi-protocol nature which helps in labeling and passing other protocols, which includes ATM across an MPLS network. MPLS signaling protocols help in establishing, maintaining and releasing label-switched paths (LSP).

\section{$5 \quad$ MPLS Features / Advantages}

The multipoint connectivity needs to be addressed in the global economy; it has clearly become necessary to connect dispersed offices, resources and stake holders geographically. International connectivity can be introduced by the help of MPLS connectivity by using high speed data service customers, MPLS can used to share mission critical data which can be secured at high levels of security and provides scalability.

\section{Five main features of the MPLS network include:}

1. Scalability: MPLS networks are specifically designed for highly scalable solutions. Only one provider edge (PE) is required to peer with the customer site as compared to all the other CPE or customer edge (CE) routers. VPNs can be created in the Layer 3 due to this connectionless architecture, it also eliminates the need for tunneling and thus scaling is improved as compared 
to the customer based VPNs, this also helps in reducing the setting up and management requirements.

2. Security: Connectionless VPNs offer the level of security as compared to that of connection oriented VPNs. Packets do not cross involuntarily and at the edge of network security is provided in order to ensure that the packets received are placed on the correct VPN. Spoofing is nearly impossible as the VPN traffic is normally kept separate. VPN labels are provided to identify particular interface and sub interfaces.

3. Quality of Service (QoS): Quality of Service and the Network performance for selected traffic can be improved using the MPLS. Different types of services bring in multiple classes of service. For example, an enterprise's mission-critical application can be placed in the top most section of service; the applications less important might be in the Second best level of service, recreational applications may be in the lower class services, separate class of service can be given to reduce the jitter for VoIP traffic. Customers can prioritize the traffic needs according to the business requirements. Packets can also be marked in order to differentiate between the high quality packets and the low end to end latency packets for voice and video.

4. Traffic Engineering: Traffic load on various links, switches and routers in the network can be balanced by routing the data traffic, this process is known as Traffic Engineering. Congestion is controlled as it has the ability to control specific routes. MPLS is capable of full traffic engineering.

5. Choice of Network Architectures: Depending on the requirements the network can be designed in various ways such as Partially Meshed, Fully Meshed, hub \& Spoke etc. Ingress and Egress routers only distinguish the different protocols.

\section{MPLS VS ATM Performance Comparison}

Traffic routing is one of the most important challenge faced for having an efficient network. Percentage of Real Time Traffic has increased over the internet due to the growing usage of Real Time Traffic applications. The core components play a very important role in enhancing the network. Due to delay sensitivity, minimum Qos requirements this kind of traffic requires more care.

MPLS and ATM both have special features which can support real time traffic. A number of new features are being introduced in IP technology in order to support the real time traffic because it is almost impossible to change the already existing IP based network and Internet. 
We use the following metrics for comparison and analysis of MPLS and ATM:

$>$ Delay (sec): It represents the end to end delay. It is the summation of all delays discussed in previous section.

$>$ Throughput (pps): represents the total number of packets forwarded to higher layers per second.

$>$ Utilization: It represents the percentage of the consumption of an available channel bandwidth where a value of 100.0 would indicate full usage.

$>$ FTP Download Response Time(sec): This is the time between the request for channel is made and when the system starts download file.

$>$ Normalized Delivered Traffic: It is the number of correctly received packets normalized by sent packets from all clients. It indirectly shows the percentage of the dropped traffic over the network during simulation. (4)

\section{$7 \quad$ Simulation Results and Analysis}

\section{i. Throughput}

It depends on the link speed and the nature of the technology being used to transmit the data. The result shows that the throughput performance for both are degraded, it can be due to two possible reasons. First, there is no Quality of Service enabled and there could be congestion along the barrier. Secondly, the degradation could be due to the packet drop along the way from Source to Destination.

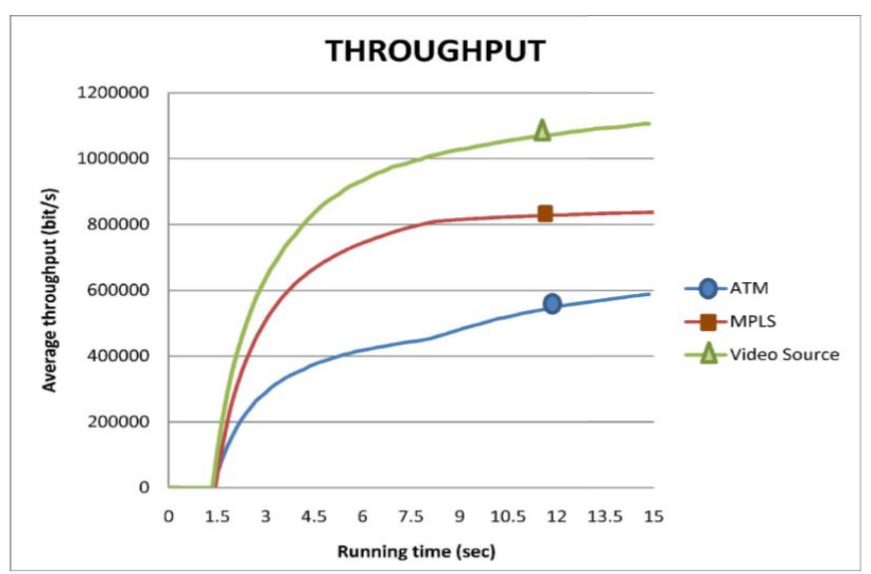

Fig. 3. Throughput Graph of MPLS and ATM (5) 


\section{ii. Queuing Delay}

The quality of the real time traffic, especially the voice and Data is destroyed due to the delay and the behavior of ATM shows that it is not fit for real time traffic. MPLS has longer average queuing delay as compared to ATM because the packet processing in MPLS node is faster than in ATM.

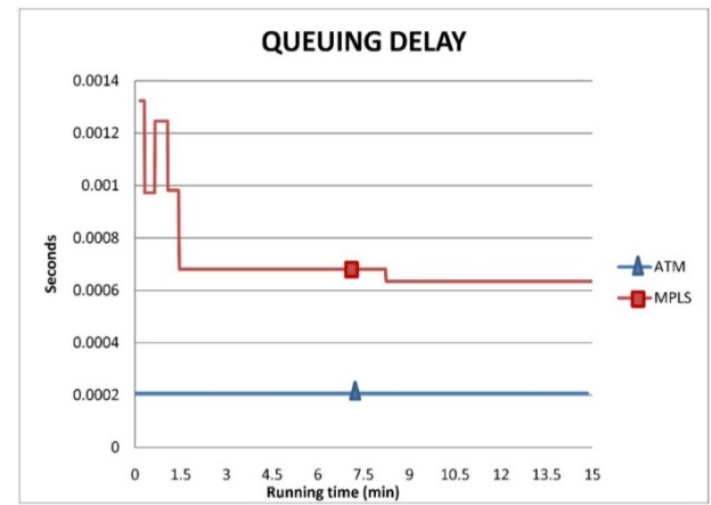

Fig. 4. Queuing Graph of ATM and MPLS (5)

\section{iii. Utilization}

Utilization is very important in order to check the efficiency of a technology. I t shows how efficiently the resources have been used. Utilization increases linearly with load until the saturation point is reached. The average bandwidth for MPLS is $41 \%$ and ATM is $28 \%$.

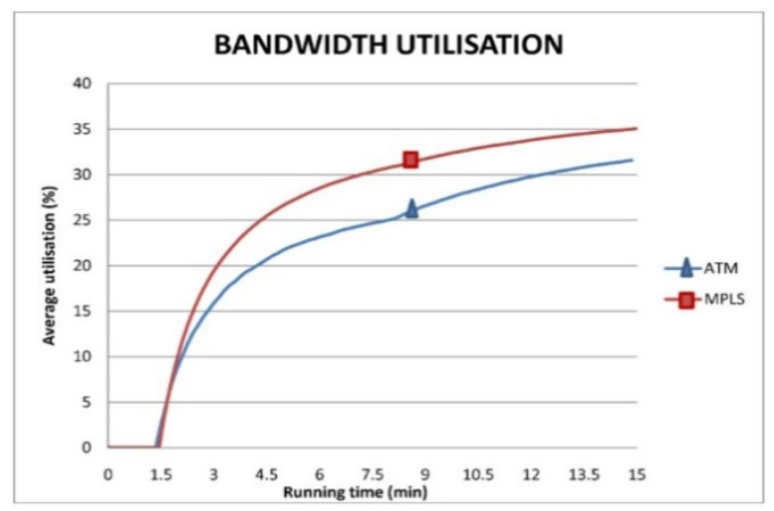

Fig. 5. Bandwidth Utilisation ATM and MPLS (5) 


\section{iv. FTP Download Response Time}

ATM response time is considerably less as compared to MPLS. This concludes that ATM gives better performance as compared to MPLS.

\section{v. Normalized Traffic Received}

Connectionless and Connection oriented effects can be seen. Connectionless behavior is responsible for the drops at the time of congestion; it also runs the congestion avoidance mechanism which helps in avoiding congestion. Connection oriented reduces dropped traffic to nil.

\section{$8 \quad$ MPLS Drawbacks}

i. One of the potential drawbacks of MPLS is that the carrier plays a very important role. In static routing the service provider is responsible for the routing whereas in dynamic routing the service provider and you will have to work together to route the traffic.

ii. MPLS security is also one pf the potential drawbacks, MPLS networks do not provide any kind of inbuilt security services which leads the network exposed to different kinds of vulnerabilities.

\section{Conclusion}

If one is looking to upgrade a network then MPLS is the way to go. MPLS is more helpful as it has the capability to combine the intelligence of routing and the performance in networking which also helps in providing a considerable amount of benefit to the network. In this paper we highlight the importance of MPLS and the need to implement it in order to overcome the limitations provided by ATM. It also explains the various standards used and also provides an insight to the performances based in MPLS networks. 


\section{References}

1. Kocak, C., Erturk, I., \& Ekiz, H. (2009). MPLS over ATM and IP over ATM methods for multimedia applications. Computer Standards \& Interfaces, 31(1), 153160. doi:10.1016/j.csi.2007.11.003

2.

3. Hyeong Ho Lee, Bu Ihl Kim, Jae Sup Lee, \& Chu Hwan Yim. (n.d.). Structures of an ATM switching system with MPLS functionality. Seamless Interconnection for Universal Services. Global Telecommunications Conference. GLOBECOM'99. (Cat. No.99CH37042). doi:10.1109/glocom.1999.830124

4. Student. Paper presented at University of Derby, Derby.

5. Bin Ali, Z., Samad, M., \& Hashim, H. (2011). Performance comparison of video multicasting over Asynchronous Transfer Mode (ATM) \& Multiprotocol Label Switching (MPLS) networks. 2011 IEEE International Conference on System Engineering and Technology. doi:10.1109/icsengt.2011.5993445 